
\title{
$\begin{array}{ll}\text { Research Square } & \begin{array}{l}\text { Preprints are preliminary reports that have not undergone peer review. } \\ \text { They should not be considered conclusive, used to inform clinical practice, } \\ \text { or referenced by the media as validated information. }\end{array}\end{array}$
}

\section{Genome-Wide Identification of PEBP Gene Family Members In Potato and Their Phylogenetic Relationships and Expression Patterns Under Heat Stress}

Guodong Zhang

Gansu Agricultural University

Xin Jin

Gansu Agricultural University

Xiubao Li

Rizhao Academy of Agricultural Sciences

Ning Zhang

Gansu Agricultural University

Shaoqian Li

Qingdao Customs of People's Republic of China

\section{Huaijun Si}

Gansu Agricultural University

Om P. Rajora

University of New Brunswick

Xiu-Qing Li ( $\sim$ Xiu-Qing.Li@agr.gc.ca)

Agriculture and Agri-Food Canada Fredericton Research and Development Centre https://orcid.org/0000-0002-7308-0596

Research Article

Keywords: Solanum tuberosum L., high temperature stress, PEBP gene family analysis, PEBP motifs, expression patterns, abiotic stress response

Posted Date: August 19th, 2021

DOI: https://doi.org/10.21203/rs.3.rs-809879/v1

License: @) (1) This work is licensed under a Creative Commons Attribution 4.0 International License. Read Full License

Version of Record: A version of this preprint was published at Molecular Biology Reports on April 2nd, 2022. See the published version at https://doi.org/10.1007/s11033-022-07318-z. 


\section{Abstract}

The phosphatidy ethanolamine-binding protein (PEBP) genes are involved in regulating plant flowering and tuberization. We analyzed both the recently updated, long-reads-based reference genome (DM v6.1) and the previous short-reads-based annotation (PGSC DM v3.4) of the potato reference genome and characterized heat-induced gene expression using RT-PCR and RNA-Seq. Fifteen PEBP genes were identified from DM v6.1 and named StPEBP1 to StPEBP15 based on their chromosomal locations. Six of these genes were not found in the previous annnotation (DM v3.4). The 15 genes could be classified into FT, TFL, MFT, and PEBP-like subfamilies and were located on 6 chromosomes. Most of the StPEBP genes were found to have conserved motifs 1 to 5 similar to Arabidopsis and other plants. We found that heat stress induced opposite expression patterns of certain FT and TFL members in a tissue-specific way: StPEBP14 and StPEBP15 versus StPEBP3 and StPEBP10 in leaves, StPEBP4 versus StPEBP10 in roots, and StPEBP9 versus StPEBP3 in tubers (FT versus TFL respectively). This maintenance of the FT/TFL opposite expression pattern but involving tissue-specific PEBP members may partly explain why different potato organs have different sensitivities to heat stress. Our study provided important multiuse genomic resource, and relevant information and candidate genes for genetic improvement of heat tolerance in potato. It clearly support that the long-reads-based genome assembly and annotation provides a better genomic resource for identification of PEBP and perhaps other genes.

\section{Introduction}

Climate change has increased the frequency and extent of heat stress conditions for plants and animals, including crop plants. Potato (Solanum tuberosum) plants are very susceptible to heat stress, which can induce many changes in plant growth, especially tuber growth $[1,2]$. Plant morphological structure, flowering, and various other biological processes including seasonal gene expression patterns are known to be regulated by phospholipid ethanolamine binding protein (PEBP) gene family members[3-6]. Therefore, identification and characterization of PEBP gene family members is necessary for understanding functional plant biology and effects of abiotic stress on plants.

The PEBP gene family, generally divided into three (FT for flowering locus T, TFL for terminal flower and MFT for mother of FT and TFL) subfamilies [7, 8], has been identified in various plants, including Arabidopsis thaliana [9], maize (Zea mays) [10], rice (Oryza sativa) [11], tomato (Lycopersicum esculentum) [12, 13], wheat (Triticum spp). [14], Chrysanthemum [15] and blueberry (Vaccinium sp.) [16]. Some studies also identified a PEBP-like subfamily in plants [17, 14]. StSP6A, a PEBP gene in potato, is a FT-like gene, which likely plays a key role in the initiation of tuber formation and tuber expansion in potato [18]. StCEN1, which belongs to the TFL subfamily, is associated with the signaling of abscisic acid and cytokinin, and regulates the bud growth rate after the release of tuber dormancy in potato [19]. Overexpression of StCEN1 can also inhibit tuber formation [20]. According to quantitative reverse-transcription polymerase chain reaction (qRT-PCR) analysis of certain PEBP genes, heat-caused changes in PEBP gene expression appeared to delay flowering in chrysanthemum [15] and it is likely responsible, to a certain degree, for the inhibition of potato tuberization [21]. Therefore, identification and characterization of PEBP gene family members and their expression patterns, especially in response to heat stress, in potato is important for understanding potato's response to climate change conditions. It is still unclear which members of the PEBP genes are most active in major organs (such as leaves, roots, and tubers) of the potato plants. Root system are very important for potato plant performance under heat stress but there is very little information available for comparative expression activities of PEBP genes in roots, regardless of whether under ambient or heat stress conditions.

Phylogenetic relationship of PEBP genes among various plant species including potato was reviewed [12], but the potato genes did not have an annotated potato gene number (PGSC, for potato genome sequence consortium) and seemed from a preliminary version of the potato reference genome [22] and the review did not report the method used to identify these genes[12]. Furthermore, the previous version of the potato genome was assembled using short reads and represented only $86 \%$ of the $844 \mathrm{mb}$ - genome of homozygous doubled-monoploid potato clone [22]. Now, an updated version (DM v6.1) of the potato genome of the same doubled-monoploid clone DM1-3 516 R44 has become available in 2020, which is based on the Oxford Nanopore Technologies long reads coupled with proximity-by-ligation scaffolding, yielding a chromosome-scale assembly [23]. Thus, genome-wide analysis of this new version of the potato genome is required to identify all of the PEBP genes and to compare them with the PEBP genes reported previously.

In the present study, we analyzed the protein database (proteins for all coding sequences and representative proteins) of the new genome version (DM v6.1) and the previous version (DM v3.4), and compared the PEBP genes identified from the gene annotation in both the genome versions as well as with the potato PEBP genes reported in the literature. The purpose was to identify the members of the PEBP gene family in potato from the updated potato reference genome [23], characterize their phylogenetic relationships/classification and conserved motifs, and analyze their gene expression patterns in different tissues in response to heat stress.

\section{Materials And Methods Plant Material}

The plantlets of Potato Cultivar 'Atlantic' was used in the qRT-PCR analysis at the Gansu Agricultural University. The cultivar 'Russet Burbank' was used for the whole transcriptome analysis of both leaves and tubers of potato plants under heat stress at the Fredericton Research and Development Centre, Agriculture and Agri-Food Canada, Fredericton, Canada.

\section{Identification of PEBP genes in potato}

We used Hidden Markov models (HMM) method [24] to identify PEBP gene family members from DM_1-3_516_R44_potato.v6.1.hc_gene_models.pep.fa of the DM v6.1 potato genome. The target protein domain was PBP, and the Pfam database number was 'PF01161'[25]. In order to improve the accuracy of the identification, we used the new annotation data DM v6.1 of the potato genome (http://solanaceae.plantbiology.msu.edu)[23], and searched for potential family members from the potato protein sequence files using the hmmsearch tool. The sequences of potential PEBP proteins were submitted to SMART[26] 
and NCBI CDD (https://www.ncbi.nlm.nih.gov/cdd/) to search for the PBP domains. PEBP family members with the Pfam PBP domain were used for further analysis.

The annotation information from the SolTub_3.0.44 version of genome annotation has been used for a long time, and many previous studies and open databases are based on this version. Therefore, we also analyzed the PGSC_DM_v3.4_pep_representative.fasta (amino acid sequences corresponding to the representative CDS file) and the PGSC_DM_v3.4_pep.fasta (amino acid sequences corresponding to all gene coding sequences) of the DM v3.4 annotation version of the DM v4.04 potato reference genome. We identified the PEBP gene family members in the PGSC gene database of the SolTub_3.0.44 genome annotation by using the same method as outlines above.

All the PEBP genes identified from the annotation information of the potato genome version DM v6.1 were named based on their chromosome locations and added "St" prefix representing Solanum tuberosum. The protein sequences of the identified members of the PEBP gene family were submitted to Expasy website(https://web.expasy.org/)to calculate theoretical PI and molecular weight [27] of the proteins coded by these StPEBP genes. UniProt[28] and STRING[29] online tools were used to annotate the identified StPEBP genes by protein sequence alignment, and KOBAS website[30] was used to perform GO and KEGG annotations.

\section{Chromosomal location, phylogenetic analysis and motif detection of the PEBP family genes in potato}

The location of the PEBP gene family members on potato chromosomes was extracted from the DM v6.1 annotation of the potato genome[23]. Then MapChart software was used to conduct mapping of these genes on individual chromosomes.

To study the divergence and evolutionary relationship of the potato PEBP genes with those from potato and other plant species, we constructed a phylogenetic tree among the protein sequences of these StPEBP genes and PEBP genes of other species, including Arabidopsis thaliana[9], rice (Oryza sativas sp. japonica)[11], apple (Malus $\times$ domestica Borkh.)[31] and tomato (Solanum lycopersicum)[12]. The PEBP protein sequences of five species including potato were aligned by Cluster W method (default parameter) using the software MEGA7 software. The Neighbor-Joining (NJ) method was used to construct a phylogenetic tree, 'test of phylogeny' as 'bootstrap method 1000', 'model / method' as 'Poisson model', 'rates along sites' as 'uniform rates' and 'gaps / missing data treatment' as 'partial deletion'.

Highly similar genes were classified into the same group/subfamily based on the phylogenetic tree grouping of the PEBP genes identified from the DM v6.1 database and the PGSC (DMV3.044 database. Each of the PEBP genes in the DM v6.1 was used to design primers for the analysis of the expression patterns of PEBP genes.

The sequences of PEBP proteins from Arabidopsis thaliana, tomato and potato were submitted to MEME website(https://meme-suite.org/)for motif analysis, using 'select the number of motifs' set to 10 , and other parameters were set as the default. The result was imported into TBtools to generate the motif distribution map.

\section{Expression analysis of PEBP family genes under both control and heat stress conditions}

We used leaves and roots of plantlets of cv Atlantic grown in hydroponic conditions to study the expression pattern of StPEBP gene family members in leaves and roots under heat stress. First, the plantlets of 'Atlantic' were transferred into glass bottles $(6 \mathrm{~cm} \times 15 \mathrm{~cm})$ and cultured on solid MS medium ( $3 \%$ sucrose). The environmental parameters were set to $22^{\circ} \mathrm{C}, 16$ hours of light per day, light intensity 10000 LUX. After 30 days of culture, the aseptically grown seedlings were transferred to the hydroponic tank containing Hoagland culture medium. In the first week of hydroponic culture, the plants were covered with transparent water cup to keep the air humidity around the plants and avoid wilting due to strong leaf transpiration. After a week-long acclimation, the water cup was removed, and the culture conditions were set to $23^{\circ} \mathrm{C}$, light intensity $10000 \mathrm{LUX}$ for 16 hours per day. Each hydroponic tank contained eight liters of Hoagland medium, and each tank contained 12 potato plants. The medium was changed every three days.

After 45 days of hydroponic culture, the seedlings were treated with high temperature. The temperature of heat stress treatment was day(light) $35^{\circ} \mathrm{C} /$ night (dark) $28^{\circ} \mathrm{C}$. The hydroponic solution was also changed on the third day of heat stress treatment. The samples were taken at Day 0 , Day 1 , Day 4 , and Day 7 after starting the heat stress treatment, and the samples of Day 0 was taken before the beginning of heat-stress treatment, which serves as plants without heat stress. The samples included fully opened functional leaves and roots. All samples contained three biological repeats, i.e., from three different potato plants. The sampled leaves were the fifth to seventh fully expanded leaf from the top to the bottom, and 'root' refers to the relatively new growing root part accounting for $1 / 2$ of the root length. The tissues were immediately wrapped with tin foil and put into liquid nitrogen for freezing of ten minutes. Then, it was stored in a $-80^{\circ} \mathrm{C}$ freezer for RNA analysis.

Transzol up kit of TransGen Biotech was used for total RNA extraction. Transcript one step gDNA removal and cDNA synthesis supermax Kit was used for cDNA synthesis. Real time quantitative PCR was used to examine the expression levels of StPBEP genes before (Day 0 , control) and after 1, 4 and 7 days of heat treatment. Real time quantitative PCR primers were designed by using primer-blast of NCBI and qPrimerDB website[32].

Real time quantitative PCR (qRT-PCR) was performed using TransStart Top Green qPCR SuperMax and Roche LightCycler 96 system. The three-step procedure was as follows: denaturation at $95^{\circ} \mathrm{C}$ for 10 seconds, annealing at $57^{\circ} \mathrm{C}$ for 20 seconds, extension at $72{ }^{\circ} \mathrm{C}$ for 30 seconds and 50 cycles were used. The $\triangle C T$ of StPEBP genes and internal reference gene in the three biological repeats were calculated and standardized $\left(2^{-\triangle C T} * 100000\right)$, and then the average value of the three biological repeats was used to represent the final expression levels of the gene.

The IBM SPSS statistics 22 was used to run One-way ANOVA and Duncan test of the qRT-PCR data from each organ (leaves and roots) for all StPEBP genes to identify which StPEBP genes were the most active. The significant level was set at $P<0.05$. Student t-test $(P<0.05$, two tails) was also used to determine 
whether the expression levels of each gene was significantly increased or decreased in comparison with the expression levels of the same gene in the same organs sampled from the Day 0 plants (immediately prior to the start of the heat treatment).

\section{Transcriptome-wide differential expression analysis of StPEBP genes in "Russet Burbank" leaves and tubers in response to heat stress}

We used the Illumina whole transcriptome sequences of the control and heat-stressed plants and tubers of cultivar Russet Burbank to examine the expression patterns of the identified StPEBP genes in response to heat stress. The $\mathrm{m}$ used for RNA extraction, library construction, Illumina sequencing and reads cleaning, mapping and DESeq test of both control plants and heat treated plants were as described previously [33]. RNA sequencing was performed using the Illumina HiSeq4000 PE-100bp platform. We conducted those studies to identify and characterize transcriptome-wide differentially expressed genes in response to heat stress. In the present study, we used that transcriptome resource for evaluating the expression patterns specifically of the StPEBP genes in response to heat stress. The unimap pipeline of TBSPG [34] was used to trim off the primers, clean the poor quality reads and map against the PGSC_DM_v4.03_transcript-update representative.fasta database. The differential gene expression analysis for the StPEBP genes was performed using DESeq [35]. The Illumina sequencing and analysis of tuber RNA has been described in detail previously [36], and the read sequences of tubers are available in the short read database of NCBI (BioProject ID:PRJNA578671).

\section{Results}

\section{Identification of the PEBP gene family in potato}

Fifteen PEBP genes (StPEBP1-StPEBP15; Table 1) were identified in the recently updated version DMV6.1 of the potato reference genome [23] using the Hidden Markov models (HMM) method [24] to search for PBP domain for PEBP family members using the Pfam database number 'PF01161'[25]. The members of these 15 StPEBP genes family were named according to their chromosomal locations, starting from Chromosome 1 (Figure 1 ). The 15 genes identified in the present study were located on chromosome 1 (three PEBP genes), 3 (three), 5 (three), 6 (one), 9 (three) and 11 (two). The protein sequences of these 15 StPEBP genes were validated by SMART(http://smart.embl.de/) and NCBI CDD (https://www.ncbi.nlm.nih.gov/cdd/) tools. SMART confirmed that the StPEBP3, StPEBP5, StPEBP6, StPEBP9, StPEBP14, StPEBP15 had the PBP domain, and NCBI CDD classified all 15 StPEBP genes from DM v6.1 to the PEBP superfamily. Theoretical isoelectric point ( $\mathrm{pl}$ ) was lowest in PEBP-like proteins (5.21 for StPEBP13; 5.53 for StPEBP12) and FT proteins (from 5.74 for StPEBP7 to 6.9 for StPEBP15), and great variation in TFL proteins (from 5.73 for StPEBP1 to 9.07 for StPEBP3) (Table 1). PEBP-like proteins had the smallest molecular weight but considerable variation was observed in the molecular weight among TFL proteins (Table 1). The nomenclature correspondence of the potato PEBP genes identified in our study to the names of potato PEBP genes published in the literature is presented in Table 1.

Table 1. Fifteen PEBP genes identified in potato 


\begin{tabular}{|c|c|c|c|c|c|}
\hline Name & $\begin{array}{l}\text { Protein ID in DM } \\
\text { v6.1(this study) }\end{array}$ & \begin{tabular}{l} 
DMP ID from \\
PGSC_DM_v3.4_pep_representative.fasta \\
『PGSC0003+\} & 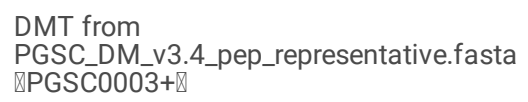 & 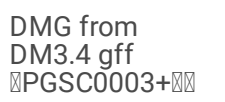 & $\begin{array}{l}\text { ID in A } \\
\text { al.[12] } \\
\text { BLAST }\end{array}$ \\
\hline StPEBP1 & Soltu.DM.01G006970.1 & & & & $\begin{array}{l}\text { Not fol } \\
74 \% \text { cc } \\
\text { Sotubc } \\
\text { CEN1 }\end{array}$ \\
\hline StPEBP2 & Soltu.DM.01G006990.1 & & & & $\begin{array}{l}\text { Sotubc } \\
\text { CEN1 }\end{array}$ \\
\hline StPEBP3 & Soltu.DM.01G007030.1 & DMP400020755 & DMT400030575 & DMG400011707 & $\begin{array}{l}\text { Sotubc } \\
\text { CEN1 }\end{array}$ \\
\hline StPEBP4 & Soltu.DM.03G011110.1 & & & & $\begin{array}{l}\text { Sotubc } \\
\text { SP3D }\end{array}$ \\
\hline StPEBP5 & Soltu.DM.03G017110.1 & DMP400025227 & DMT400037143 & DMG400014322 & $\begin{array}{l}\text { Sotubc } \\
\text { CEN1 }\end{array}$ \\
\hline StPEBP6 & Soltu.DM.03G033490.1 & DMP400009953 & DMT400014409 & DMG400005654 & $\begin{array}{l}\text { Sotubc } \\
\text { MFT }\end{array}$ \\
\hline StPEBP7 & Soltu.DM.05G024030.1 & & & & $\begin{array}{l}\text { Sotubc } \\
\text { SP5G- }\end{array}$ \\
\hline StPEBP8 & Soltu.DM.05G024040.1 & & & & $\begin{array}{l}\text { Sotubc } \\
\text { SP5G-1 }\end{array}$ \\
\hline StPEBP9 & Soltu.DM.05G026370.1 & DMP400040404 & DMT400060057 & DMG400023365 & $\begin{array}{l}\text { Sotubc } \\
\text { SP6A }\end{array}$ \\
\hline StPEBP10 & Soltu.DM.06G029780.1 & DMP400012606 & DMT400018307 & DMG400007111 & $\begin{array}{l}\text { Sotubc } \\
\text { SP/TF }\end{array}$ \\
\hline StPEBP11 & Soltu.DM.09G003550.1 & DMP400062201 & DMT400090526 & DMG400040097 & $\begin{array}{l}\text { Sotubc } \\
\text { SP9D }\end{array}$ \\
\hline StPEBP12 & Soltu.DM.09G008810.1 & DMP400011109 & DMT400016032 & DMG400006267 & No fou \\
\hline StPEBP13 & Soltu.DM.09G008890.1 & & & & No fou \\
\hline StPEBP14 & Soltu.DM.11G004040.1 & DMP400028268 & DMT400041725 & DMG400016179 & $\begin{array}{l}\text { Sotub1 } \\
\text { SP5G-1 }\end{array}$ \\
\hline StPEBP15 & Soltu.DM.11G004050.1 & DMP400028269 & DMT400041726 & DMG400016180 & No fou \\
\hline
\end{tabular}
\end{tabular}

Note: The 15 StPEBP genes identified from sequence analysis and annotation of the DM v6.1 reference genome http://solanaceae.plantbiology.msu.edu/dm_v6_1_download.shtml and were named according to their chromosomal locations. DMT and DMP IDs were from the PGSC protein sequences of PGSC_DM_v3.4_pep_representative.fasta (of the DM v3.4 version of the potato reference genome). DM_1-

3_516_R44_potato.v6.1.hc_gene_models.pep.fa were also analyzed but results were identical to the analysis of the representative proteins except all protein sequence database had some duplicates. Gene ID with 'Sotub' were according to Abelenda et al. 2014[12]; The correspondence between the StPEBP 'Gene ID in SolTub ID' were identified using NCBI-blastn; 'pl' means theoretical isoelectric point, 'MW' means Molecular weight [27]. The enes names starting with Sotub- were according to 'Function': the annotation information and were confirmed by both Uniprot analysis and STRING analysis of protein sequences. 'GO annotation' and 'KEGG annotation': annotation information from KOBAS[30]. 'PEBP-like' in Function: Phosphatidylethanolamine binding protein'. StPEBP12, StPEBP13 and StPEBP15 of the present study were not in the 15 gene list of Abelenda et al. 2014[12] according to BLASTn comparison (no significant similarity

Comparison between the 15 PEBP genes identified in the present study and the 15 PEBP genes annotated and labelled as PEBP genes in the potato DM v6.1 reference genome

We searched the DM v6.1 reference potato genome using http://solanaceae.plantbiology.msu.edu/ with the keyword "PEBP". Among the15 items found by searching the reference genome (Table S1), we found that that the Soltu.DM.11G004050.2 was on the list of genes, but it is from the alternative splicing of Soltu.DM.11G004050 of the same gene PGSC0003DMG400016180 ( StPEBP15 gene). The StPEBP10 and StPEBP11 genes were not annotated as PEBP genes in the DM v6.1 reference genome but labelled as centroradialis (for CEN gene in or list) despite these genes had the PBP domain. Therefore, these two genes should be classified as PEBP genes, as we did in the present study. The "Soltu.DM.01G007000.1" gene was annotated and labeled as a PEBP gene in the DM v6.1 potato genome, and this gene was picked up as a PEBP gene when we searched the DM v6.1 with the keyword "PEBP". However, we did not identify this gene as a PEBP gene because it does not contain a PBP domain (Table S1). 
\#: Searched the DM v6.1 using http://solanaceae.plantbiology.msu.edu/ with the keyword "PEBP". Among the genes found by searching the reference genome, the Soltu.DM.11G004050.2 was on the list of genes found by the search, but it is from the alternative splicing of Soltu.DM.11G004050 of the same gene PGSC0003 DMG400016180, the StPEBP15 gene.

Comparison between the PEBP genes identified by us from the DM v6.1 reference genome and from the DM v3.4 reference genome

From the analysis of the protein sequences of the PGSC_DM_v3.4_pep_representative.fasta file, we identified nine PEBP proteins, including PGSC0003DMP400020755 for StPEBP3, and PGSC0003DMP400028269 for StPEBP15 (please see the full list in Table 1). These nine genes corresponded to StPEBP3, StPEBP5, StPEBP6, StPEBP9, StPEBP10, StPEBP11, StPEBP12, StPEBP14, StPEBP15 genes of the present study. Six StPEBP genes (StPEBP1, StPEBP2, StPEBP4, StPEBP7, StPEBP8, StPEBP13) were found in the DM v6.1 gene/protein sequence database but not in the DM v3.4 gene/protein sequence database. We also analyzed the PGSC_DM_V3.4_pep.fasta database and classified 10 transcripts/proteins as PEBP, but two of the transcripts (PGSC0003DMT400037142 and PGSC0003DMT400037143) or proteins (PGSC0003DMP400025226 and PGSC0003DMP400025227) were from the same gene (PGSC0003DMG400014322). Therefore, only 9 PEBP genes were annotated as PEBP genes in the version DM v3.4 reference genome and 6 PEBP genes were not annotated in that former version.

\section{Comparison between the PEBP genes identified in the present study and the 15 PEBP genes in the literature}

Four of the 15 StPEBP genes (StPEBP1, StPEBP12, StPEBP13, and StPEBP15; Table 1) identified in the present study were novel compared to the genes in the previous study [12]. In the BLASTn search for these four different genes: StPEBP1 had only 74\% coverage with Sotub016190 CEN1 [12] ; StPEBP12 and StPEBP13 did not correspond directly to any of the former 15 PEBP genes [12] ; and the StPEBP15 had only $79.82 \%$ identity with the previous gene Sotub11g010050 [12] (Table 1).

\section{KEGG and gene ontology (GO) analysis of the 15 StPEBP genes identified in the present study}

The KEGG analysis identified that StPEBP4, StPEBP7, StPEBP8, StPEBP9, StPEBP14 and StPEBP15 genes are related to the circadian rhythm of plants (sot04712, circadian rhythm - plant). GO annotation identified StPEBP3 and StPEBP5 as regulators of plant flowering (GO: 0009908, flower development; GO: 0010228, vegetable to reproducible phase transition of meristem), and StPEBP6 as a regulator for ABA response and seed germination (G0: 0009737, response to abscisic acid; GO: 0010030, positive regulation of seed). And StPEBP11 was annotated to be involved in nucleus (GO: 0005634), cytoplasm (GO: 0005737), negative regulation of flower development (GO: 0009910), and vegetable to reproducible phase transition of meristem (G0: 0010228).

\section{Phylogenetic analysis and motif detection of the PEBP family genes in potato}

The neighbor-joining phylogenetic tree of 59 PEBP genes from potato, tomato, Arabidopsis, rice, and apple constructed using the MEGA7 software revealed the relationship of potato PEBP proteins in relation to those in other model plants (Figure 2). The results suggested that 15 StPEBP genes could be classified into four subfamilies (Figure 2). StPEBP4, StPEBP7, StPEBP8, StPEBP9, StPEBP14 and StPEBP15 were classified in the FT subfamily. StPEBP1, StPEBP2, StPEBP3, StPEBP5, StPEBP10, and StPEBP11 were closely related to AtTFL1 (TFL subfamily). StPEBP6 was closely related to AtMFT and could be classified in the MFT subfamily. StPEBP12 and StPEBP13 were far away from the other StPEBP genes in the phylogenetic tree, and they were classified into PEBP-like subfamily (Figure 2). This is consistent with the annotation results of UniProt and STRING.

The phylogenetic tree constructed based on 25 StPEBP protein sequences from potato, 15 from the DM v6.1 data and 10 from the SolTub_3.0.44 data (Figure 3) clarified the relationships between the genes/gene names in the present study and those in the previous version SolTub_3.0.44. StPEBP1 1 STPEBP3, and StPEBP5 were found to be homologous to PGSC0003DMT400030582, PGSC0003DMT400030575, and PGSC0003 DMT400037143هrespectively. These three genes were annotated as StCEN1. StPEBP10, and StPEBP11 were found to be close to GSC0003DMT400018307, and StSP9D (T90526), respectively. StPEBP2 was partly homologous to PSC0003DMT400030575. All of these six genes (StPEBP1,StPEBP5,StPEBP2, StPEBP3, StPEBP10, and StPEBP11) are members of the TFL subfamily. StPEBP4, StPEBP9, StPEBP7, StPEBP8, StPEBP14, and StPEBP15 genes were found belonging to the FT subfamily, and the closest homologues were FLOWERING LOCUS T protein (PGSC0003DMT400060057هSTSP6A) and FLOWERING LOCUS T (PGSC0003DMT400041725, PGSC0003DMT400041726). StPEBP6 of the MFT subfamily was found to be homologous to StMFT (PGSC0003DMT400014409). StPEBP12 and StPEBP13 were closely related to phosphatidylethanolamine binding protein (PGSC0003DMT400016032).

\section{Motif detection of the potato PEBP family genes}

Motif analysis of 34 PEBP proteins from Arabidopsis thaliana囚tomato and potato using MEME online tools (https://meme-suite.org/) identified a total of 10 motifs (named as No. 1-10; Table S2). We found that each PEBP gene contains 3-6 motifs. Eight StPEBP genes (StPEBP4, StPEBP45, StPEBP47, StPEBP48, StPEBP49, StPEBP410, StPEBP414, and StPEBP415) had five motifs, motif 1, 2, 3, 4 and motif 5. StPEBP2 and StPEBP3 had motif s1-5 and motif 7, and StPEBP6 consist of motifs 1-5 and motif 10. StPEBP1 had motifs 1, 3, 4, 5, 7 and motif 10 . StPEBP11 had motifs 1, 2, 4, and 5 (Table 1).

Note: the sequence of 34 PEBP proteins were submitted to the MEME website (https://meme-suite.org/) for motif identification. Ten motifs were conserved in PEBP genes of potato, tomato, and Arabidopsis thaliana.

All six Arabidopsis thaliana PEBP proteins (AtPEBP) are composed of motifs 1, 2, 3, 4, and 5. The structure of eight tomato PEBP proteins was similar to that of the AtPEBP proteins in Arabidopsis. Two tomato CEN1 genes (Solyc01009580 Solyc01009560) contained motifs 1-5 and motif 7, while other three FT-like tomato PEBP genes (Solyc05055660, Solyc11008650, Solyc11008660) contained three or four motifs (Figure 4). 
In real-time quantitative reverse transcriptase polymerase chain reaction (qRT-PCR) analysis of the expression levels of StPEBP genes in leaves using their specific primers (Table S3) identified relative activities (expression levels) of the genes in leaves and which genes were differentially expressed between Day 0 (control) and treated plants (Table 2). The cDNA sequences were too similar to have separate specific primers between StPEBP2 and StPEBP3 (both were TFL genes), between StPEBP7 and StPEBP8 (both were FT genes), and between StPEBP12 and StPEBP13 (both were PEBP-like genes). None of these three groups of similar genes showed high activities from the qRT-PCR analysis in leaves (Table 2). At Day 0, the expression of StPEBP15 in leaves was most active among the 15 StPEBP genes. StPEBP14 was the second most active gene in leaves. The expression level of StPEBP11 and StPEBP12 (StPEBP13 together) was too low $(C T>35)$ to be detected both for Day 0 control leaves and Day 1 and Day 4 of heat-stressed leaves (Table 2).

Note囚UBI-F, UBI-R are primers of housekeeping gene used as the internal control[41]. The StPEBP1, 2, 6, 7, 13 had no specific qRT-PCR primers designed because qPrimerDB [32] could not generate specific qRT-PCR regions from the specific regions of their cDNA sequences. The primer pair StPEBP3-F and StPEBP3-R could amplify both StPEBP2 and StPEBP3 (both are TFL genes). Primers for StPEBP8 could also amplify StPEBP7 (both are FT genes). Primers for StPEBP12 can also amplify StPEBP13 (both are PEBP-like genes).

Table 2. The expression levels of PEBP genes in heat stressed mature leaves and roots of potato

\begin{tabular}{|c|c|c|c|c|c|c|c|c|}
\hline \multirow[t]{2}{*}{ Gene Name } & \multicolumn{4}{|c|}{ Expression levels in leaves } & \multicolumn{4}{|c|}{ Expression levels in roots } \\
\hline & Day 0 & Day 1 & Day 4 & Day 7 & Day 0 & Day 1 & Day 4 & Day 7 \\
\hline StPEBP3 & $1.2 \mathrm{f}$ & \# & \# & $14.7 f * \star$ & $23.5 \mathrm{dc}$ & $12.2 \mathrm{dc}$ & $16.1 \mathrm{dc}$ & $35.8 d-b$ \\
\hline StPEBP4 & $3.9 \mathrm{f}$ & $\#$ & \# & $5.03 \mathrm{f}$ & $9.4 \mathrm{dc}$ & $5.0 \mathrm{dc}$ & $5.7 \mathrm{dc}$ * & $8.4 \mathrm{dc}$ \\
\hline StPEBP5 & $59.2 \mathrm{f}$ & $240.8 \mathrm{f}$ & $\#$ & $95.1 \mathrm{f}$ & $7.3 \mathrm{dc}$ & $17.9 \mathrm{dc}$ & $22.8 \mathrm{dc}$ & $36.5 d-b$ \\
\hline StPEBP8 & $88.3 \mathrm{f}$ & $76.4 \mathrm{f}$ & $142.3 \mathrm{f}$ & $191.9 f *$ & $18.5 \mathrm{dc}$ & $39.5 d-b$ & $19 \mathrm{dc}$ & $39.7 d-b$ \\
\hline StPEBP9 & $5.4 \mathrm{f}$ & \# & \# & $4.8 \mathrm{f}$ & $5.5 \mathrm{dc}$ & \# & $6.8 \mathrm{dc}$ & $10.1 \mathrm{dc}$ \\
\hline StPEBP10 & $11.2 \mathrm{f}$ & $27.7 \mathrm{f}$ & \# & $75.5 f *$ & $14.7 \mathrm{dc}$ & $22 \mathrm{dc}$ & $72.4 \mathrm{~b}$ * & $293.1 \mathrm{a}$ * \\
\hline StPEBP11 & \# & \# & \# & $1.96 \mathrm{f}$ & $35.8 d-b$ & $5.3 \mathrm{dc}$ & $18.4 \mathrm{dc}$ & $47.9 \mathrm{cb}$ \\
\hline StPEBP12 & $\#$ & \# & \# & $1.89 \mathrm{f}$ & $4.5 \mathrm{dc}$ & $4.9 \mathrm{dc}$ & $3.7 \mathrm{dc}$ & $10.4 \mathrm{dc}$ \\
\hline StPEBP14 & $1571.1 \mathrm{e}$ & $145.1 \mathrm{f} * \star$ & $50.6 f * *$ & $405.3 f \star \star$ & $10.8 \mathrm{dc}$ & $16 \mathrm{dc}$ & $7.1 \mathrm{dc}$ & $17.1 \mathrm{dc}$ \\
\hline StPEBP15 & $8793.9 \mathrm{a}$ & $3517.6 d * *$ & $4863.5 c^{* *}$ & 7708.6 b & $7.1 \mathrm{dc}$ & $2.3 \mathrm{~d}$ & $5.7 \mathrm{dc}$ & $13.5 \mathrm{dc}$ \\
\hline
\end{tabular}

Note囚\#: means the gene activity was too low to be detected and its Ct value of qRT-PCR is greater than 35. "StPEBP3" primers may pick transcripts of StPEBP1, StPEBP2 and StPEBP3 genes because too high similarity among these three sequences. Similar situation existed between StPEBP7 and StPEBP8 and also between StPEBP12 and StPEBP13. 'Expression' represents the average value of $2^{-\triangle C T} \times 100000$ of three biological replicates. One-way ANOVA was followed by Duncan's multiple range test, with the $5 \%$ significance level; the expression of leaves and roots were analyzed separately. *: The expression level was significantly different from Day 0 of the same gene in the same organ. The values followed by different letters in a row for a gene are significantly differently expressed $(P<0.05)$.

Under heat stress, both StPEBP14 and StPEBP15 genes were down-regulated on all Day 1, Day 4, and Day 7, but StPEBP3 (StPEBP2), StPEBP7/StPEBP8 (formerly StSP5G genes) and StPEBP10 genes were up-regulated in potato leaves (Table 2) on Day 7. StPEBP15 was still the most active gene in leaves among all genes under heat stress even through its activity was down-regulated (Table 2). In our transcriptome-wide analysis of leaves of the 'Russet Burbank' plants using the second-generation sequencing (Illumina), heat stress deceased the expression activities of StPEBP5, StPEBP9, StPEBP14, and StPEBP15 (Table S4).

Note: '\#'means the difference in expression level is not significant.'RB1' is according to our previous study on the expression level in 'Russet Burbank' leaves after three days of heat [33]; 'Agria' gene expression is according to the study in leaves of the whole plant under heat stress [42]; 'Desiree' gene expression is according to the gene expression level of leaves [21]; 'RB2' is according to our previous study on heat stressed 'Russet Burbank' tubers (the Illumina read sequences are available in NCBI BioProject ID:PRJNA578671; under publication elsewhere).

\section{Expression patterns of StPEBPs in heat-stressed roots}

In the control plants, activities of all 10 genes or groups of genes that had specific primers designed were detectable by qRT-PCR in the roots (Table 2). The gene StPEBP11, whose expression was not detectable in leaves, was the most active StPEBP gene in roots (Table 2).

Under heat stress conditions in roots, the expression of StPEBP10 was increasingly up regulated with the prolongation of stress, and it reached the peak on Day 7 of the heat treatment. The gene StPEBP4 was found to be downregulated on Day 4 after the starting of the heat treatment. The expression of other StPEBP genes was still detectable in heat-stressed roots but heat stress did not induce significant changes (Table 2).

\section{Expression patterns of StPEBP genes in heat-stressed tubers}

In the heat-stressed tuber transcriptome of the potato cultivar 'Russet Burbank', DESeq analysis showed that the expression of StPEBP9 (PGSC0003DMT400060057, formerly StSP6A) appeared to be mildly downregulated in response to heat stress (log2fc $=-1.5)($ Table S4). We did not find 
other StPEBP genes to be differentially expressed in response to heat stress in the whole transcriptome.

\section{Discussion}

Identification of StPEBP genes detected considerable differences between two versions (DM v3.4 and DM v6.1) of the potato genome annotations. We have identified 15 StPEBP genes in potato based on the latest potato genome annotation, DM v6.1 version

(http://solanaceae.plantbiology.msu.edu/dm_v6_1_download.shtml, visited on April 20,2021). Only 9 of these 15 genes were found in the PGSC annotations (PGSC transcripts, DMT, or protein DMP or genes DMG). Furthermore, only 11 of the 15 PEBP genes we identified in the latest version of the potato genome were found previously in potato based on the literature review. It was unknown how the 15 potato PEBP were identified previously [12], but only seven of them were officially annotated in the DMT, DMP databases of the annotation version 3.4 of the potato reference genome DM v3.04 and DM v4.04

(http://solanaceae.plantbiology.msu.edu/pgsc_download.shtml) (Table 1). Our results demonstrate that compared to DM v6.1, approximately 33\% (5/15) of the PEBP genes were absent in the DM v3.4 annotation of the reference genome of the same potato doubled monoploid DM genotype. Our comparative genomic analysis of the PEBP gene family provides a surprising example that third generation long-read sequencing apparently is more powerful than the combined approach of BAC library and Illumina second generation sequencing for genome decoding.

The StPEBP genes identified in the present study are more nearly complete because we identified several PEBP genes not found in the previous genome versions, removed false PEBP genes (no PBP domain) (Table S1), and removed duplicated transcripts from the same genome. Therefore, the 15 StPEBP genes identified in the present study likely represent a more accurate repertoire of StPEBP genes to date. It is a coincidence that the DMP (DM potato proteins annotated by PGSC) number was all 15 from following three search or analysis approaches: a) searching with the key word "PEBP", b) the present study from the analysis of DM v6.1, and the previous reports [12], and c) the present study from the analysis of the all-cds proteins database of DM v3.4 annotation (Table 1). It appears that the long-reads-based genome assembly and annotation provides a better genomic resource for identification of PEBP and perhaps other genes. We believe that this inconsistency in different genome versions is due to the differences in genome assembly and annotation between the two versions because two different sequencing technologies and platforms were used in two versions of the potato genome [12]. The reference genome (DMv6.1) [23] published in 2020 is based on long reads and has a more complete and comprehensive annotation of the potato genome. Therefore, the 15 StPEBP genes we identified from the DMv6.1 annotation are likely more representative of the actual PEBP genes in the DM potato clone. These genes provide an excellent genomic resource for various research and applications, especially understanding how the PEBP genes influence various traits in potato.

The phylogenetic analysis of 59 PEBP genes from potato, tomato, Arabidopsis, rice and apple, and evolutionary relationships between StPEBPS and Arabidopsis $P E B P$ genes, suggest that 15 StPEBP genes can be grouped into four subfamilies: FT, TFL, MFT and PEBP-like. We suggest that StPEBP4, StPEBP7, StPEBP8, StPEBP9, StPEBP14 and StPEBP15 genes belong to the FT subfamily. StPEBP7, StPEBP8, StPEBP14 and StPEBP15 were found to be close to $S P 5 G$ and $S P 5 G$-like genes of tomato and can be annotated as SP5G-like genes of potato. It is generally believed that StSP5G can inhibit tuber formation by regulating the expression of StSP6A[12]. On the other hand, StPEBP4 is close to tomato SP3D, and the SP3D protein (or SFT protein) of tomato leaves can be transported to the shoot apical meristem (SAM) through phloem, and then form florigen activation complex (FAC) with 14-3-3 and SPGB protein[12]. It is also known that StSP3D has a similar flowering function[18]. Our study indicates that StPEBP9 is highly similar to SISP6A and StSP6A (PGSC0003DMT400060057) (Fig. 2 and Fig. 3). Out results suggest that StPEBP1, StPEBP2, StPEBP3, StPEBP5, StPEBP10 and StPEBP11 genes belong to the TFL subfamily, and StPEBP6 gene belongs to the MFT subfamily. We found StPEBP6gene to be genetically close to AtMFT. Our results demonstrate that StPEBP12 and StPEBP13 genes are far away from the other StPEBP genes in the phylogenetic tree; thus, we identified these two genes as members of the PEBP-like subfamily (Fig. 2). PEBPlike genes may perform functions different from other PEBP genes and are likely involved in photoperiod response and regulation of flowering in different cotton genotypes in cotton [17]. Little is known of the PEBP-like genes in potato. We also found that the conserved motifs of the PEBP-like proteins are very different from that of other PEBP genes. The differences in motifs may provide some clue to the function of PEBP-like genes. Nevertheless, our study probably provides the first evidence for the existence of PEBP-like genes in potato.

Gene motif analysis in our study demonstrates that five motifs (motifs 1-5) are conserved in all except two PEBP genes in potato. The same motifs are also found in most of the PEPB genes in other plants. All of six PEBP genes in Arabidopsis thaliana have the same motifs 1-5 (Fig. 4). More than 50\% of tomato PEBP proteins also have the same motifs (Fig. 4). AtFT,SISP3D and StPEBP4, homologous to SISP3D, which are part of FAC(Flowering Activation Complex)[12] also have motifs $1-5$. This indicates that motifs $1-5$ are the most common conserved sequence of the PEBP proteins and perhaps the structural basis for the PEBP proteins to exert their biological functions, especially to regulate flowering. StSP6A (PGSC0003DMT400060057) is an important gene to induce tuber formation[12], and StPEBP9 gene is homologous to StSP6A gene (Fig. 3). The potato StPEBP9 gene in our study contains all conserved motifs $1-5$ but the tomato SISP6A (Solyc5g055660) gene was found to have only motifs1, 3, 4, and 5, missing motif 2 . This suggests that motif 2 may play a key role in the process of tuber formation. The motif 7 was found only in STPEBP1, StPEBP2, and StPEBP3 genes, which was annotated as StCEN1 and two tomato CEN1 genes (Solyc01g009580, Solyc01g009560). This result suggests that motif 7 may be involved in the core functional components of StCEN1 proteins to repress tuberization.

Gene expression analysis in the present study found that StPEBP14 and StPEBP15, two FT-like genes, were the most active genes, about a thousand-fold more active than StPEBP9 (former name: StSP6A) in leaves of young potato plants under control conditions (Table 2). The results may suggest that StPEBP14 and StPEBP15 play an important role in receiving environmental signals and are involved in regulating potato plant developmental stages. Transgenic and/or gene editing studies may help determine the biological functions of these two genes.

Heat stress considerably changed the expression patterns of PEBP genes in leaves and detected an opposite expression patterns of some FT and TFL genes: Down-regulated two FT genes (StPEBP14 and StPEBP15) but up-regulated two TFL genes (StPEBP3 and StPEBP10) (Table 2). These results are consistent with our previous transcriptome analysis of leaves of cv Russet Burbank under heat stress [33]. In tomato, SISP5G and SISP5G-like are floral inhibitors and their expression are controlled by the photoperiod [27]. The potato StPEBP14 (a FT gene) and StPEBP15 (a FT gene) are homologous of SISP5G and SISP5G-

Page $8 / 13$ 
like (Fig. 2), whereas, StPEBP10 (a TFL gene) is likely a negative regulator for flowering according to GO analysis (G0:0009910: negative regulation of flower development). StPEBP10 is also found to be homologous of SITFL1(Solyc06g074350 SP/TFL1)which performs as floral inhibitor and has a role in sympodial shoot architecture[12]. Arabidopsis FT is a flowering promoter, but the AtTFL 1 plays as a flowering repressor[18]. StCEN10T37143[can repress tuber formation, whereas StSP6AIPGSC0003DMT400060057ロis the core inducer of tuberization [20]. Within the FT gene group, the downregulation of StPEBP14 and StPEBP15 was associated with upregulation of StPEBP8 suggests there might be a activity switch among FT gene members under heat stress (Table 2). Further research is required to investigate what roles these opposite expression patterns of StPEBP14 and StPEBP15 versus StPEBP3, StPEBP8, and StPEBP10 play for flower induction and tuberization under heat stress.

Root gene expression analysis of the present study found that heat stress in roots induced also an opposite expression patterns between FT and TFL genes but different gene members from the FT/TFL pair in leaves. In leaves, the opposite FT versus TFL pairs were StPEBP14 and StPEBP15 versus StPEBP3 and StPEBP10. In roots, heat stress downregulated FT StPEBP4 gene and upregulated TFL StPEBP10 gene on Day 4 of heat treatment in potato roots (Table 2). Unlike leaves that had very high expression activities of StPEBP14 and StPEBP15, Roots were found to have detectable expression activities of 10 StPEBP genes under both non-stressed and heat-stressed conditions, but none of them were highly expressed (Table 2). It is known that heat stress has negative effect on root growth and root activity[37,38]. This gene expression pattern in the present study is in agreement that FT-like genes and TFL-like genes usually have the antagonistic functions in plant growth and development[39]. Further research is required for the roles of this opposite expression patterns between these TFL and FT genes on root growth and heat tolerance of the potato plants. In our study, the expression of TFL gene StPEBP10 was up-regulated significantly in potato roots; therefore, we deduced that StPEBP10 may play a role in regulating root development under heat stress.

Tuber gene expression under heat stressed also showed a decrease of a FT gene (StPEBP9) and increase of a TFL gene (StPEBP3) (Table S4). Interestingly, we discovered that heat stress downregulated different FT gene members in different organs: StPEBP14 and StPEBP15 in leaves, StPEBP4 in roots, and StPEBP9 in tubers. Our results of the gene expression profiling at the whole transcriptome level in the control and heat-stressed potato tubers of cv Russet Burbank suggest mild downregulation of StPEBP09 gene in response to heat stress in potato tubers. Microarray-based analysis of heat- stressed tubers of the cultivar 'Desiree' by Hancock et al. [21] also found that heat-stress caused down-regulation of this gene (fold change was - 2.6) (Table S4). Considering the transcriptome data from these two independent experiments, we tentatively conclude that most of the StPEBP genes do not dramatically change their expression levels in potato roots and tubers in response to heat stress.

\section{Conclusion}

We have identified 15 StPEBP genes in potato; six of which are novel in terms of the comparison with previous genome versions (v3.4, PGSC), and four were novel in terms of the comparison with the published information in plants. The present study on PEBP gene family supports that the long-reads-based genome assembly and annotation provides a better genomic resource for identification of PEBP and perhaps other genes. The 15 StPEBP genes belong to FT, TFL, MFT and PEBP-like four sub-families of the genes and are located on six chromosomes. Most of the StPEBP genes have conserved motifs 1 to 5 , similar to Arabidopsis and other plants. However, the conserved motifs of the StPEBP genes belonging to potato PEBP-like proteins were quite different from that of other PEBP genes, suggesting potentially distinct roles of the PEBP-like genes in potato. We have identified the StPEBP genes active in leaves and roots of potato plants and differentially expressed genes in response to heat stress in potato leaves, roots, and tubers. A pattern of FT gene downregulation associated with TFL gene upregulation was detected in both leaves and roots under heat stress but are different gene members are involved in these organs, suggest tissue-specific differentiation of PEBP members while expression patterns between gene groups was maintained. In heat stressed leaves, FT genes StPEBP14 and StPEBP15 were downregulated but TFL genes StPEBP3 and StPEBP10 were upregulated. In roots, FT StPEBP4 was downregulated, whereas TFL gene StPEBP10 was upregulated in response to heat stress. In tubers, FT StPEBP9 was downregulated but TFL gene StPEBP3 was upregulated. Our results on the $P E B P$ gene family, motif identification, phylogenetic classification and expression patterns under heat-stress in potato enhance the knowledge about potato $P E B P$ genes and provided candidate genes for various genomics studies in potato, including genetic engineering of potato plant for heat tolerance.

\section{Declarations}

\section{Acknowledgements}

This research was supported by National Natural Science Foundation of China (No. 31860399 to Huaijun Si), Natural Sciences and Engineering Research Council of Canada Discovery Grant (RGPIN 2017-04589) to Om P. Rajora, and the Agriculture and Agri-Food Canada support to Xiu-Qing Li in Research and Development Centre, AAFC, Government of Canada. We thank the China Scholarship Council of the P. R. China for the financial support to Guodong Zhang, a PhD student under the Joint PhD training program between Agriculture and Agri-Food Canada and Education Ministry of P.R. China.

\section{Author contributions}

GZ and HS designed the study and drafted the manuscript, GZ and XJ conducted the analysis and experiments, XL, NZ, SL, HS, OPR and XQL contributed to discussion and data interpretation, OPR and XQL revised and reshaped the manuscript and contributed to the overall direction of the manuscript. All authors approved the manuscript.

\section{Conflict of interest statement}

The authors declare no conflict of interest.

\section{References}


1. Tang R, Niu S, Zhang G, Chen G, Haroon M, Yang Q, Rajora OP, Li X-Q (2018) Physiological and growth responses of potato cultivars to heat stress. Botany 96:897-912. http://dx.doi.org/10.1139/cjb-2018-0125

2. Zhang G, Tang R, Niu S, Si H, Yang Q, Bizimungu B, Regan S, Li XQ (2020) Effects of earliness on heat stress tolerance in fifty potato cultivars. Am J Potato Res 97:23-32. 10.1007/s12230-019-09740-9

3. Karlgren A, Gyllenstrand N, Källman T, Sundström JF, Moore D, Lascoux M, Lagercrantz U (2011) Evolution of the PEBP gene family in plants: functional diversification in seed plant evolution. Plant physiology 156:1967-1977

4. Panjama K, Suzuki E, Otani M, Nakano M, Ohtake N, Ohyama T, Bundithya W, Sueyoshi K, Ruamrungsri S (2019) Isolation and functional analysis of FLOWERING LOCUS T orthologous gene from Vanda hybrid. J Plant Biochem Biotechnol 28:374-381. 10.1007/s13562-019-00487-2

5. Yu X, Liu H, Sang N, Li Y, Zhang T, Sun J, Huang X (2019) Identification of cotton MOTHER OF FT AND TFL1 homologs, GhMFT1 and GhMFT2, involved in seed germination. PloS one 14:e0215771

6. Nose M, Kurita M, Tamura M, Matsushita M, Hiraoka Y, Iki T, Hanaoka S, Mishima K, Tsubomura M, Watanabe A (2020) Effects of day length- And temperature-regulated genes on annual transcriptome dynamics in Japanese cedar (Cryptomeria japonica D. Don), a gymnosperm indeterminate species. PLoS ONE 15. 10.1371/journal.pone.0229843

7. Hedman H, Källman T, Lagercrantz U (2009) Early evolution of the MFT-like gene family in plants. Plant Mol Biol 70:359-369

8. Adeyemo OS, Hyde PT, Setter TL (2019) Identification of FT family genes that respond to photoperiod, temperature and genotype in relation to flowering in cassava (Manihot esculenta, Crantz). Plant reproduction 32:181-191

9. Kobayashi Y, Kaya H, Goto K, Iwabuchi M, Araki T (1999) A pair of related genes with antagonistic roles in mediating flowering signals. Science 286:1960-1962

10. Danilevskaya ON, Meng X, Hou Z, Ananiev EV, Simmons CR (2008) A genomic and expression compendium of the expanded PEBP gene family from maize. Plant physiology 146:250-264

11. Chardon F, Damerval C (2005) Phylogenomic analysis of the PEBP gene family in cereals. J Mol Evol 61:579-590

12. Abelenda JA, Navarro C, Prat S (2014) Flowering and tuberization: a tale of two nightshades. Trends Plant Sci 19:115-122

13. Borovsky Y, Mohan V, Shabtai S, Paran I (2020) CaFT-LIKE is a flowering promoter in pepper and functions as florigen in tomato. Plant Sci 301. 10.1016/j.plantsci.2020.110678

14. Dong L, Lu Y, Liu S (2020) Genome-wide member identification, phylogeny and expression analysis of PEBP gene family in wheat and its progenitors. PeerJ 8:e10483

15. Nakano Y, Higuchi Y, Sumitomo K, Oda A, Hisamatsu T, Naro (2015) Delay of flowering by high temperature in chrysanthemum: heat-sensitive time-of-day and heat effects on CsFTL3 and CsAFT gene expression. The Journal of Horticultural Science Biotechnology 90:143-149

16. Gaire R, Wilde HD (2018) Natural allelic variation in blueberry TERMINAL FLOWER 1. Plant Genetic Resources: Characterisation Utilisation 16:59-67. $10.1017 / \mathrm{S} 1479262116000435$

17. Zhang X, Wang C, Pang C, Wei H, Wang H, Song M, Fan S, Yu S (2016) Characterization and functional analysis of PEBP family genes in upland cotton (Gossypium hirsutum L.). PLoS ONE 11:e0161080

18. Navarro C, Abelenda JA, Cruz-Oró E, Cuéllar CA, Tamaki S, Silva J, Shimamoto K, Prat S (2011) Control of flowering and storage organ formation in potato by FLOWERING LOCUS T. Nature 478:119-123. http://dx.doi.org/10.1038/nature10431

19. Morris WL, Alamar MC, Lopez-Cobollo RM, Castillo Cañete J, Bennett M, Van der Kaay J, Stevens J, Kumar Sharma S, McLean K, Thompson AJ (2019) A member of the TERMINAL FLOWER 1/CENTRORADIALIS gene family controls sprout growth in potato tubers. J Exp Bot 70:835-843

20. Zhang X, Campbell R, Ducreux LJ, Morris J, Hedley PE, Mellado-Ortega E, Roberts AG, Stephens J, Bryan GJ, Torrance L, Chapman SN, Prat S, Taylor MA (2020) TERMINAL FLOWER-1/CENTRORADIALIS inhibits tuberisation via protein interaction with the tuberigen activation complex. Plant J 103:22632278

21. Hancock RD, Morris WL, Ducreux LJ, Morris JA, Usman M, Verrall SR, Fuller J, Simpson CG, Zhang R, Hedley PE (2014) Physiological, biochemical and molecular responses of the potato (Solanum tuberosum L.) plant to moderately elevated temperature. Plant Cell Environ 37:439-450

22. The Potato Genome Sequencing C (2011) Genome sequence and analysis of the tuber crop potato. Nature 475:189. 10.1038/nature10158 https://www.nature.com/articles/nature10158\#supplementary-information

23. Pham GM, Hamilton JP, Wood JC, Burke JT, Zhao H, Vaillancourt B, Ou S, Jiang J, Buell CR (2020) Construction of a chromosome-scale long-read reference genome assembly for potato. GigaScience 9. 10.1093/gigascience/giaa100

24. Eddy SR (2004) What is a hidden Markov model? Nature biotechnology 22:1315-1316

25. Wang M, Tan Y, Cai C, Zhang B (2018) Identification and expression analysis of phosphatidy ethanolamine-binding protein (PEBP) gene family in cotton. Genomics

26. Letunic I, Khedkar S, Bork P (2021) SMART: recent updates, new developments and status in 2020. Nucleic Acids Res 49:D458-D460

27. Artimo P, Jonnalagedda M, Arnold K, Baratin D, Csardi G, De Castro E, Duvaud S, Flegel V, Fortier A, Gasteiger E (2012) ExPASy: SIB bioinformatics resource portal. Nucleic acids research 40:W597-W603

28. Consortium U (2015) UniProt: a hub for protein information. Nucleic acids research 43:D204-D212

29. Szklarczyk D, Gable AL, Lyon D, Junge A, Wyder S, Huerta-Cepas J, Simonovic M, Doncheva NT, Morris JH, Bork P (2019) STRING v11: protein-protein association networks with increased coverage, supporting functional discovery in genome-wide experimental datasets. Nucleic acids research 47:D607D613 
30. Xie C, Mao X, Huang J, Ding Y, Wu J, Dong S, Kong L, Gao G, Li CY, Wei L (2011) KOBAS 2.0: a web server for annotation and identification of enriched pathways and diseases. Nucleic Acids Res 39:W316-W322. 10.1093/nar/gkr483

31. Kotoda N, Hayashi H, Suzuki M, Igarashi M, Hatsuyama Y, Kidou S-i, Igasaki T, Nishiguchi M, Yano K, Shimizu T (2010) Molecular characterization of FLOWERING LOCUS T-like genes of apple (Malusx domestica Borkh.). Plant and cell physiology 51: 561-575

32. Lu K, Li T, He J, Chang W, Zhang R, Liu M, Yu M, Fan Y, Ma J, Sun W (2017) qPrimerDB: a thermodynamics-based gene-specific qPCR primer database for 147 organisms. Nucleic acids research 46:D1229-D1236

33. Tang R, Gupta SK, Niu S, Li XQ, Yang Q, Chen G, Zhu W, Haroon M (2020) Transcriptome analysis of heat stress response genes in potato leaves. Mol Biol Rep 47:4311-4321. 10.1007/s11033-020-05485-5

34. Xiang H, Li X-Q (2016) Development of TBSPG pipelines for refining unique mapping and repetitive sequence detection using the two halves of each Illumina sequence read. Plant Mol Biol Rep 34:172-181. 10.1007/s11105-015-0912-8

35. Anders S, Huber W (2010) Differential expression analysis for sequence count data Genome Biol. 11: R106-R117

36. Zhang G, Tang R, Niu S, Si H, Yang Q, Rajora OP, Li X-Q (2021) Heat sprouting and associated differential gene expression in growing potato tubers: Comparative transcriptomics with that induced by postharvest sprouting. Horticulture Research 9: In press

37. Sattelmacher B, Marschner H, Kühne R (1990) Effects of root zone temperature on root activity of two potato (Solanum tuberosum L.) clones with different adaptation to high temperature. J Agron Crop Sci 165:131-137

38. Sattelmacher B, Marschner H, Kühne R (1990) Effects of the temperature of the rooting zone on the growth and development of roots of potato (Solanum tuberosum). Ann Bot 65:27-36

39. Moraes TS, Dornelas MC, Martinelli AP (2019) FT/TFL1: Calibrating Plant Architecture. Frontiers in plant science 10:97

40. Abelenda JA, Cruz-Oró E, Franco-Zorrilla JM, Prat S (2016) Potato StCONSTANS-like1 suppresses storage organ formation by directly activating the FTlike StSP5G repressor. Curr Biol 26:872-881. http://dx.doi.org/10.1016/j.cub.2016.01.066

41. Lehretz GG, Sonnewald S, Hornyik C, Corral JM, Sonnewald U (2019) Post-transcriptional Regulation of FLOWERING LOCUS T Modulates Heat-Dependent Source-Sink Development in Potato. Curr Biol 29:1614-1624.e3. 10.1016/j.cub.2019.04.027

42. Hastilestari BR, Lorenz J, Reid S, Hofmann J, Pscheidt D, Sonnewald U, Sonnewald S (2018) Deciphering source and sink responses of potato plants (Solanum tuberosum L.) to elevated temperatures. Plant Cell Environ 41:2600-2616

\section{Figures}

chr1

chr3

- StPEBP4

41501424.0

StPEBP5

57131124.0 chr5

chr6

chr9

chr11

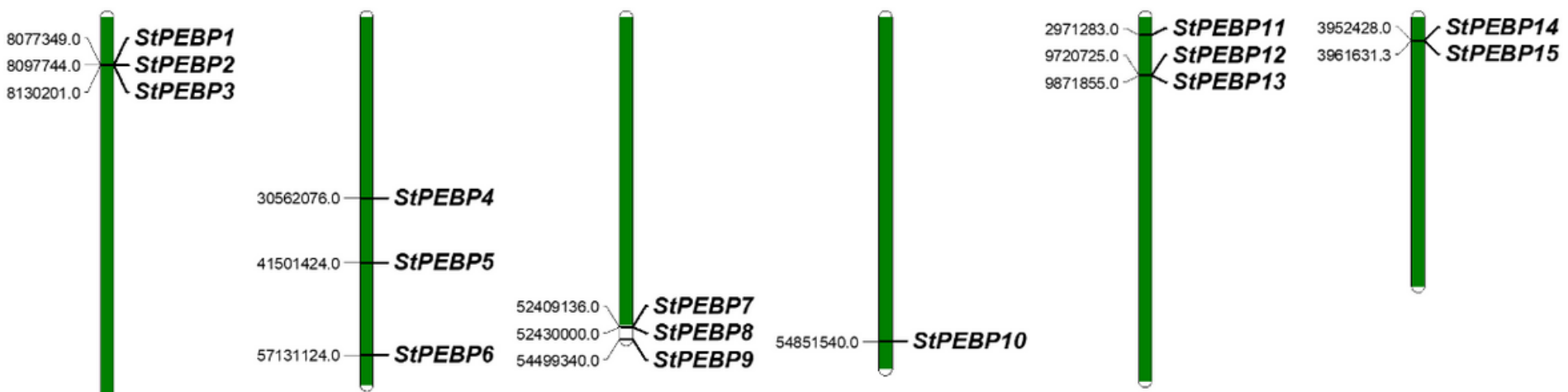

\section{Figure 1}

The chromosomal location of PEBP genes in potato (Solanum tuberosum). 


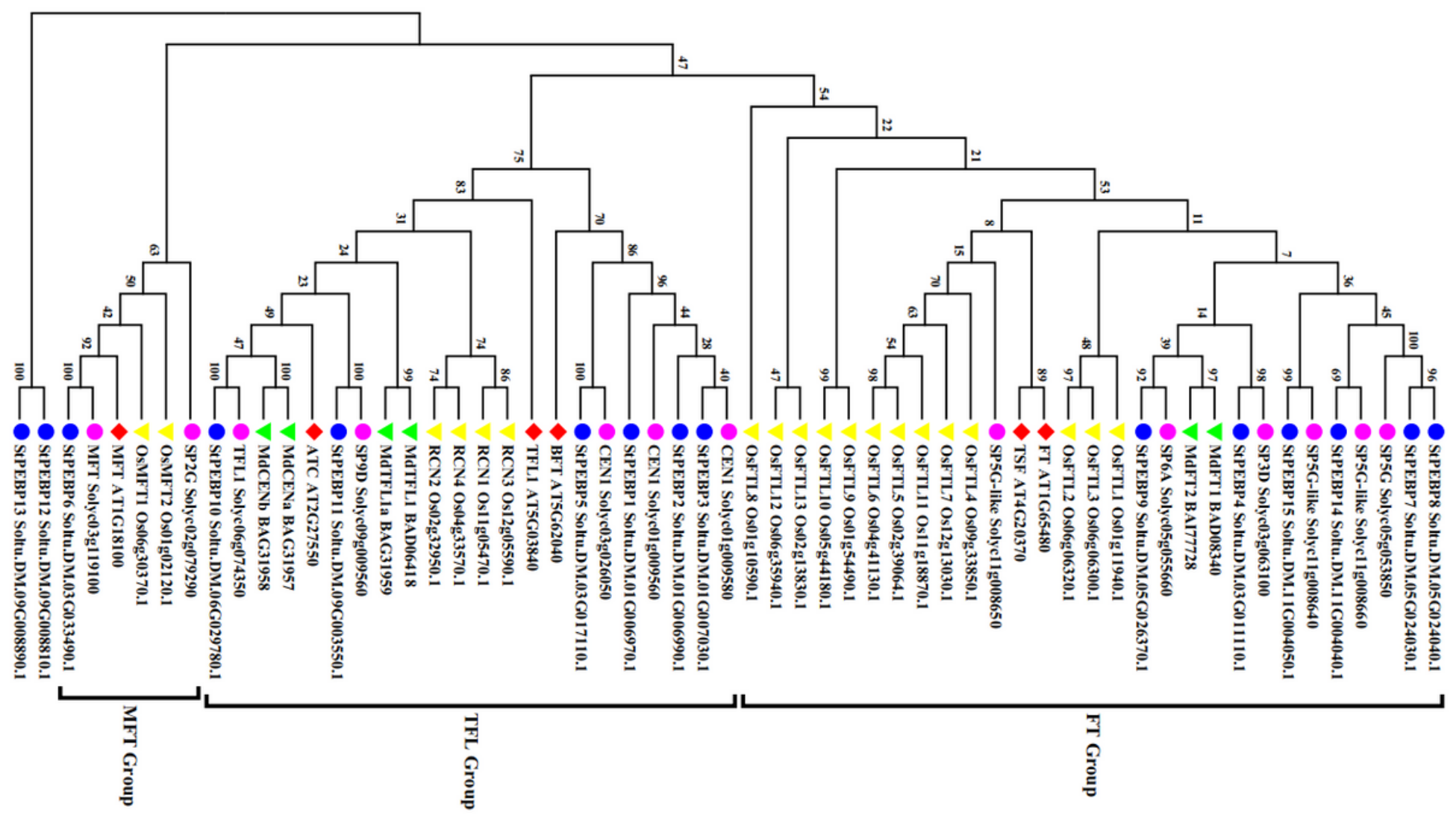

Figure 2

Neighbor-joining phylogenetic tree of 59 PEBP proteins from Arabidopsis, tomato, apple, rice and potato. Purple, blue, red, yellow and green represent genes from tomato, potato, Arabidopsis, rice, and apple, respectively.

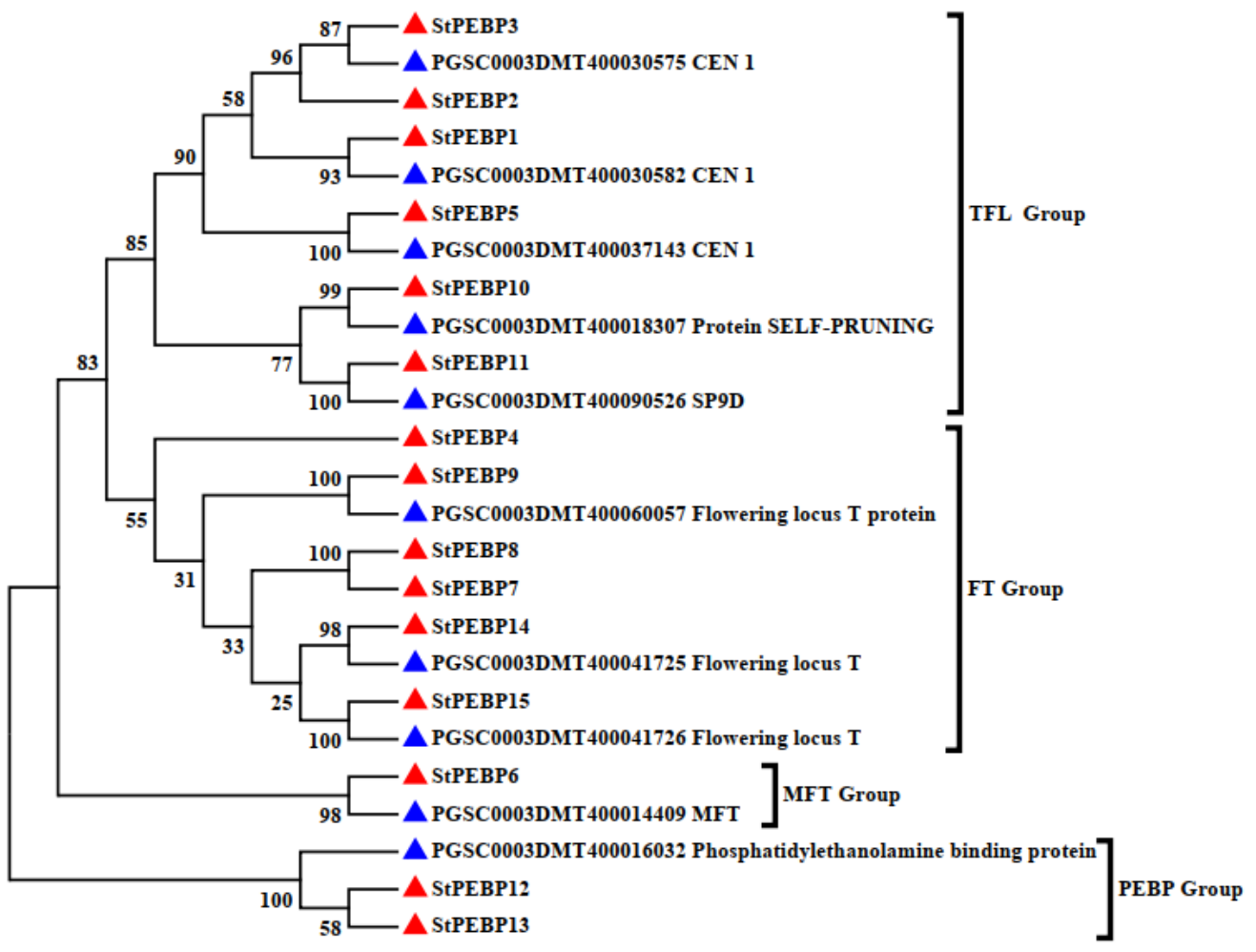

Figure 3

Neighbor-joining phylogenetic tree of 25 potato PEBP proteins to identify which DMv6.1 genome and the 10 PGSC genes from the SolTub_3.0.44 genome, with purpose to show the correspondence relationship between genes from the two version of the assembled genome sequences. Red and blue represent genes 
identified in DMv6.1 data and SolTub_3.0.44 data, respectively.

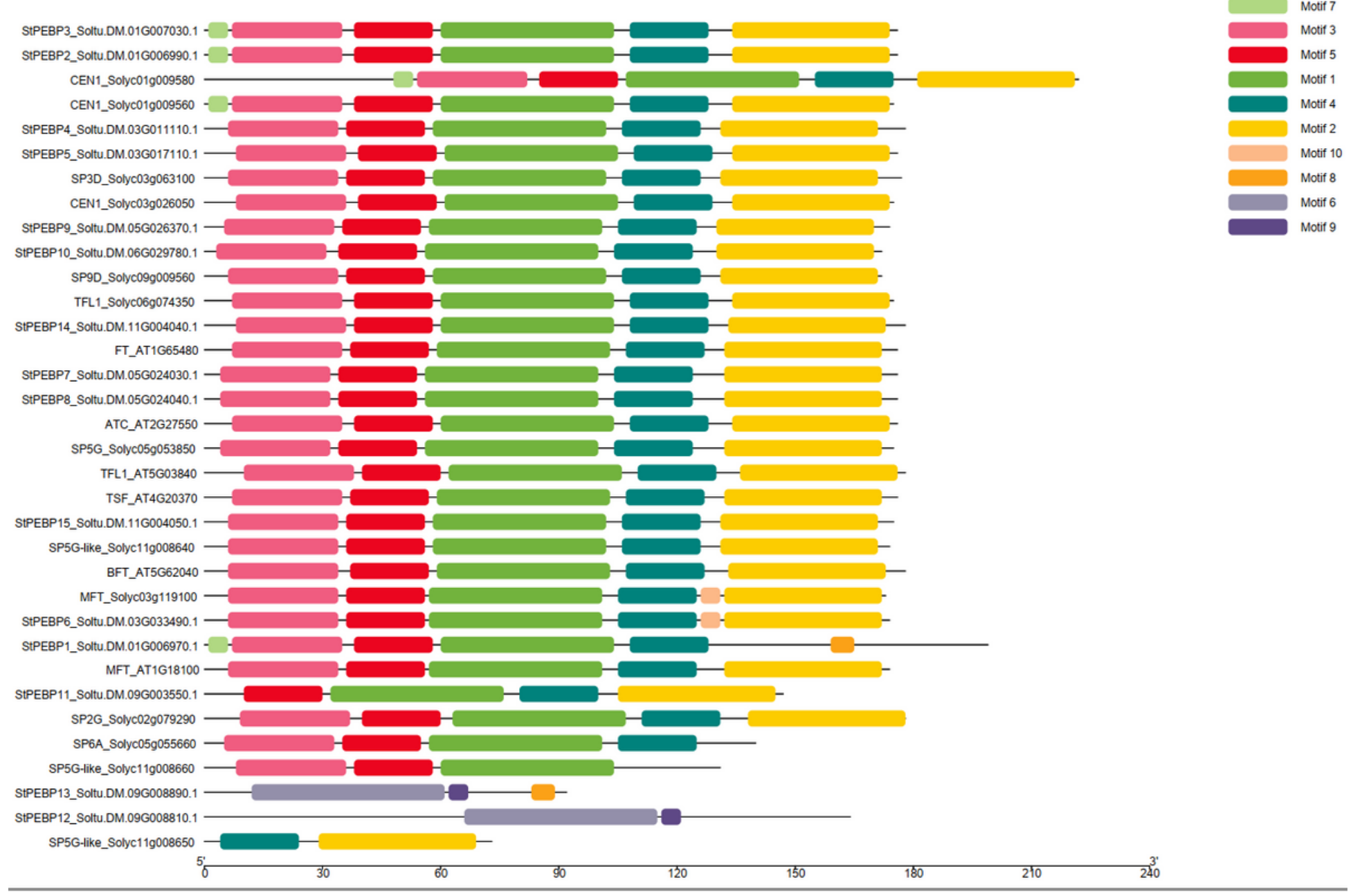

\section{Figure 4}

Motif distribution of 34 StPEBP proteins from potato, tomato, and Arabidopsis thaliana. The sequences of these 34 PEBP proteins were submitted to the MEME website (https://meme-suite.org/) for motif identification, and ten motifs (Table S2) were identified from these proteins.

\section{Supplementary Files}

This is a list of supplementary files associated with this preprint. Click to download.

- TableS.docx 